\title{
Uterine Corpus Carcinoma and Carcinosarcoma cM1 TNM Finding v8
}

National Cancer Institute

\section{Source}

National Cancer Institute. Uterine Corpus Carcinoma and Carcinosarcoma CM1 TNM

Finding v8. NCI Thesaurus. Code C139761.

Uterine corpus carcinoma or carcinosarcoma with distant metastasis (includes

metastasis to ing uinal lymph nodes, intraperitoneal disease, lung, liver, or bone. It

excludes metastasis to pelvic or para-aortic lymph nodes, vagina, uterine serosa, or adnexa). (from AJCC 8th Ed.) 